\title{
Cryogenic servo-stabilized Fabry-Perot interferometers for imaging at 3-5 and 8-13 microns.
}

\author{
N. K. Reay \& K.A.R.B. Pietraszewski \\ Queensgate Instruments Ltd., Silwood Park, Ascot, Berkshire, SL5 7PW, UK
}

\begin{abstract}
The performance of a new liquid Nitrogen cooled Fabry-Perot etalon for imaging at $3-5 \mu \mathrm{m}$ is described. Capacitance sensors monitor the etalon mirror spacing and parallelism, and error signals produced as a consequence of changes in these parameters are used in a feedback loop with piezoelectric actuators for active cavity control. These new cryogenic etalons are designed to be compatible with the Queensgate Instruments Ltd CS100/ET servo-stabilized Fabry-Perot system.

The cryogenic etalon has a clear aperture of $50 \mathrm{~mm}$ and a nominal mirror spacing of between 5 and $60 \mu \mathrm{m}$. It is coated for the $3-5 \mu \mathrm{m}$ spectral region, although coatings are also available for the $2-2.5 \mu \mathrm{m}$ and $8-13 \mu \mathrm{m}$ regions. Under servo-control at operating temperature the etalon has a response time of $30 \mathrm{msec}$ and a minimum cavity tuning range of $\pm 3 \mu \mathrm{m}$ about the nominal cavity length, corresponding to approximately 3 orders of interference at the midrange wavelength of $4 \mu \mathrm{m}$.
\end{abstract}

\section{Introduction}

The development of the scanning servo-stabilized Fabry-Perot (FP) interferometer system (Hicks, Reay \& Scaddan 1974, Hicks, Reay and Stephens 1976) utilizing capacitance micrometer sensors which detect changes in mirror parallelism, and piezoelectric (PZT) actuators for cavity tuning, has resulted in widespread use of this type of instrument in astronomy and remote sensing. Development of the system, comprising CS100 controller and ET-Series etalon, was initiated at Imperial College in 1971 by one of the authors (NKR) and continued at Queensgate Instruments, and is now in regular use at many of the World's major astronomical observatories. A derivative of the system is used in the WINDII experiment (Wind Imaging Interferometer) on the NASA - Upper Atmosphere Research Satellite (Canadian Astronautics Ltd. Document Number 376-CAL-341SP-006 Rev.1)

The success of the ground based system and the growth of airborne and satellite remote sensing applications at wavelengths of $3-5 \mu \mathrm{m}$ and $10-12 \mu \mathrm{m}$ has stimulated the development of cryogenic etalons for these wavelength regions, which are compatible with the CS100 control system. A hardware development program was initiated by Queensgate in 1986, and several liquid Nitrogen cooled etalons for $3-5 \mu \mathrm{m}$ wavelength region have now been built and tested. 


\section{The CS100 Servo-control System}

The CS100 is a three-channel, balanced bridge system which uses error signals derived from capacitance sensors, located in the etalon cavity, to control the voltages on piezoelectric actuators which correct changes in parallelism and spacing of the mirrors. The system will maintain etalon parallelism and cavity spacing to better than $0.1 \%$ of a Free Spectral Range (FSR), at a wavelength of $500 \mathrm{~nm}$, and can be computer controlled via either integral IEEE-488 or RS232 interfaces.

The capacitance sensor configuration in a standard room temperature etalon is shown in figure 1 . Three piezoelectric actuators $(\mathrm{P})$, which determine the cavity length, are cemented between the mirrors. Five capacitance sensors, $\mathrm{CX}_{1}, \mathrm{CY}_{1}$....etc., are formed by evaporating gold pads onto one of the interferometer mirrors and onto fused silica pillars optically contacted to the other mirror.

Parallelism information is obtained by comparing $\mathrm{CX}_{1}$ with $\mathrm{CX}_{2}(\mathrm{X}$ - channel) and $C Y_{1}$ with $C Y_{2}$ (Y - channel). Cavity length control is achieved by referencing $\mathrm{CZ}$ to a stable fixed reference capacitor. A summary of those aspects of system performance relating to the stability of an ambient temperature etalon is given in table 1 below: a full description of the system can be found in Hicks et al $(1974,1976)$.

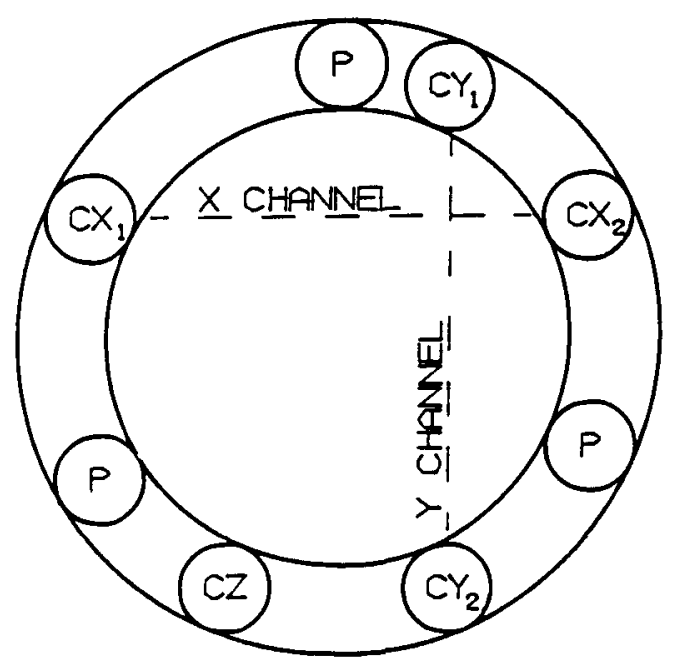

Figure 1. Schematic arrangement of the capacitors and PZT stacks in the ambient temperature ET-series etalons. The $\mathrm{X}$ and $\mathrm{Y}$ channels control parallelism, and the capacitor $\mathrm{CZ}$ is referenced to a fixed value capacitor to control mirror spacing.

Electronic noise equivalent displacement of etalon mirrors

Electronic Drift

(Temperature stability)
: $10 \mathrm{pmHz}^{-1 / 2} \mathrm{RMS}$

$: 0+/-50 \mathrm{pmC}^{-1}$

Table 1. Outline stability and scan specification for standard etalon. 


\section{The Cryogenic Etalon}

Wherever possible the design rules developed for the ambient temperature etalon were adopted for its cryogenic counterpart. Although significant changes had to be made to accommodate the $220 \mathrm{degK}$ temperature difference between assembly and operation, the basic philosophy that the etalon should be rigidly constructed was adhered to. The principal advantage of the rigid design is that the "talon is relatively immune from vibration, although this presented some difficult problems related to cementing together unlike materials, and assembling the etalon such that the correct mirror spacing and parallelism were achieved at operating temperature without the need for gross external adjustment.

\subsection{Specification}

The specification for the cooled etalon is contained in table 2 below. Additionally, it was required that it be controllable with the CS100 controller, and we should seek to achieve, insofar as it was possible to do so, the noise and stability specification in table 1.

It was also required of the design that the etalon should be capable of assembly with any nominal cavity length down to $3 \mu \mathrm{m}$. This aspect of the specification is particularly important in that cavity lengths of $3 \mu \mathrm{m}$ or so are required if the system is to function as a tunable filter at 3-5 $\mu \mathrm{m}$.

$\begin{array}{ll}\text { Operational temperature } & : 77 \text { degrees Kelvin (liquid nitrogen) } \\ \text { Mirror material } & : \text { Zinc Selenide } \\ \text { Clear aperture } & : 50 \mathrm{~mm} \\ \text { Cavity tuning range } & : \text { minimum } 1.5 \text { orders (at } 77 \mathrm{~K} \text { ) } \\ \text { Etalon finesse } & :>40 \\ \text { Transmission } & :>60 \% \\ \text { Cooling time } & :<6 \text { hours }\end{array}$

Table 2. Specification for cryogenic etalon

\subsection{Construction}

The cryogenic etalon is shown in figure 2 with the outer sleeve removed to show the internal construction. The Zinc Selenide mirrors are mounted in Invar cells, which in turn are suspended from three short PZT actuators cemented to the back surfaces of each mirror cell. The long 'external' and short 'internal' actuators operate in series and the reentrant design merely serves to reduce the overall length of the etalon whilst maintaining the required scan range.

On cooling, differential expansion between the various materials used in the etalon construction cause the mirror spacing to change, although this effect was minimized by thermally compensated design. There is also a possibility that unequal contraction of the three PZT actuators will take the parallelism of the cavity beyond the range of the servo-system. Careful analysis of the expansion loop, and cooling tests, were required to characterize this effect and enable the etalon to be assembled at ambient temperature such that on cooling it achieved the design cavity spacing and parallelism. The screw mountings were provided for the 'long' PZT actuators (see figure 2) to allow mirror parallelism and spacing to be adjusted with the etalon at ambient temperature. Once the 'correct' initial 
setup conditions were determined, ie those which on cooling resulted in an acceptable mirror spacing and parallelism, the screw joints were locked in place.

Capacitance bridge and HV connections to the CS100 are via the flying leads seen at the rear of the etalon (figure 2). A small region at the outer edge of the etalon is coated for the visible to facilitate initial alignment of the mirrors by eye; this can be seen as an aperture to the front of the top ring and mirror cell in figure 2.

\section{Performance}

Laboratory measurements were primarily concerned with testing the mirror spacing, parallelism and figure, scan range, response time and the mechanical integrity of the etalon and in particular its ability to withstand repeated cooling cycles. We were also concerned to ensure that the etalon retained the ease of use qualities of its ambient temperature counterpart; the standard ET ambient temperature etalons can be connected to the servo-system and made parallel and ready for use by setting predetermined offsets on the front panel of the CS100.

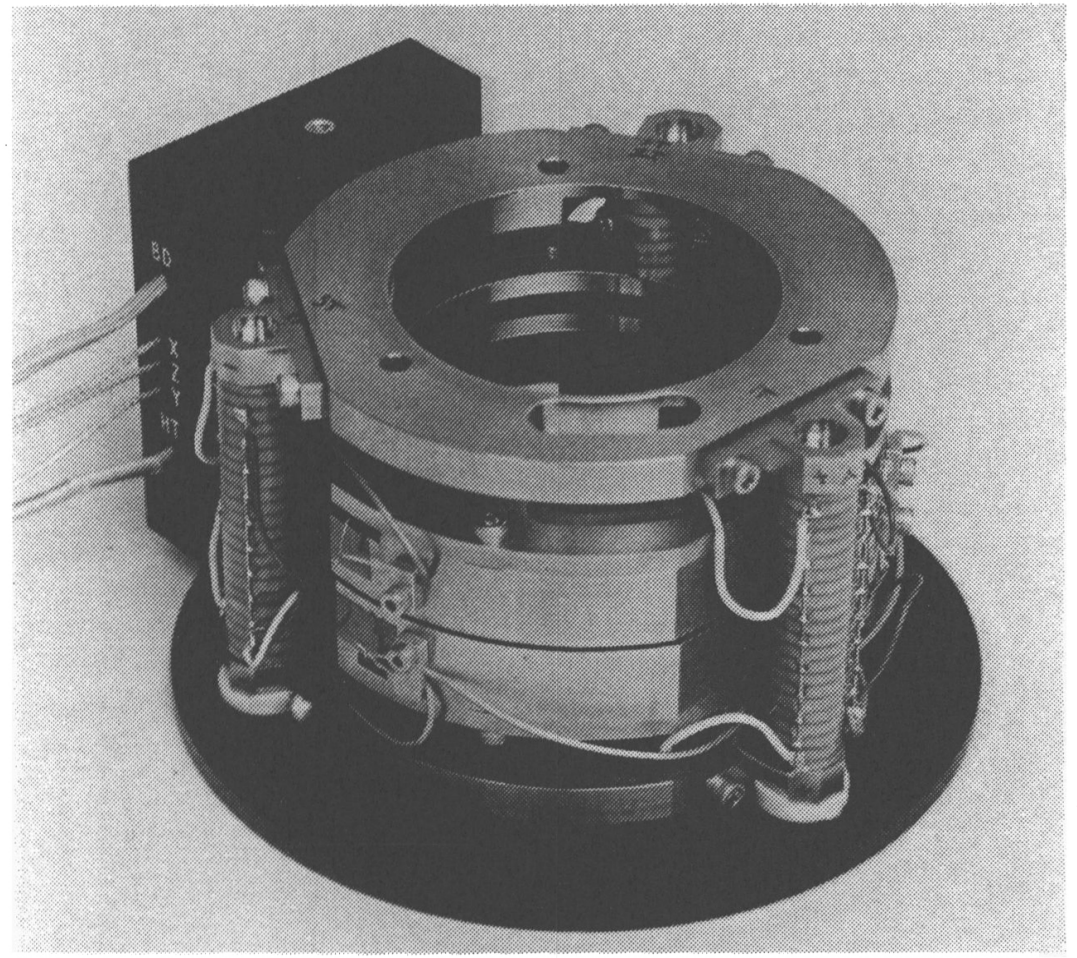

Figure 2. The Cryogenic etalon with outer sleeve removed to show the internal construction. The aperture in the top ring (forefront of photograph) allows an area of etalon coated for the visible to be viewed to facilitate initial alignment by eye. 
Table 3 compares some of the important optical and servo-loop parameters 'warm' and 'cold' for an etalon with a nominal cavity spacing of $60 \mu \mathrm{m}$.

\begin{tabular}{|c|c|c|c|}
\hline & Warm (in air) & Warm (vacuum) & Cold \\
\hline Lowest Resonant Frequency & $1.4 \mathrm{kHz}$ & $700 \mathrm{~Hz}$ & $700 \mathrm{~Hz}$ \\
\hline $\begin{array}{l}\text { Response Time (step input, } \\
\text { with CS } 100 \text { controller) }\end{array}$ & $2 \mathrm{msec}$ & $5 \mathrm{msec}$ & $30 \mathrm{msec}$ \\
\hline Mirror Spacing (nominal) & $60 \mu \mathrm{m}$ & $60 \mu \mathrm{m}$ & $55 \mu \mathrm{m}$ \\
\hline Mirror Tuning Range & $>20 \mu \mathrm{m}$ & $>20 \mu \mathrm{m}$ & $>6 \mu \mathrm{m}$ \\
\hline
\end{tabular}

Table 3. Cavity and servo-loop parameters for cryogenic etalon

The reduction in the frequency of the lowest resonance, when evacuated, is due to the reduction in air damping in the etalon cavity. As the etalon is cooled, the response time of the CS100 must be progressively increased to a value of $30 \mathrm{msec}$ at liquid Nitrogen temperature in order to avoid system oscillations. In principal the etalon is controllable with a faster response time, but the CS100 would require significant modification.

All measurements reported in the following sections were made with the etalon at liquid Nitrogen temperature.

\subsection{Mirror Reflectivity and Finesse}

Figure 3 shows a coating curve for the $3-5 \mu \mathrm{m}$ region, and figure 4 shows a finesse scan obtained by scanning the $3.39 \mu \mathrm{m}$ line from a HeNe laser with the etalon at liquid Nitrogen temperature. The finesse of 40 is consistent with the coating reflectivity of $95 \%$ and a mirror figure of $\lambda / 120$ at $3.39 \mu \mathrm{m}$.

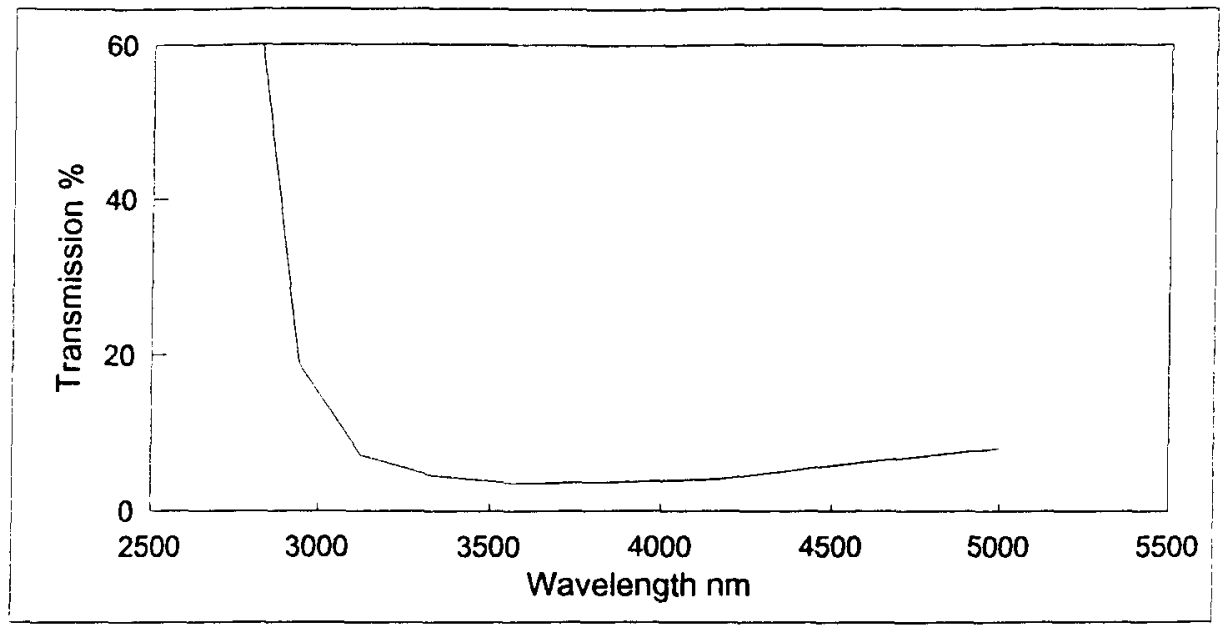

Figure 3. Coating curve for the $3-5 \mu \mathrm{m}$ wavelength region. 


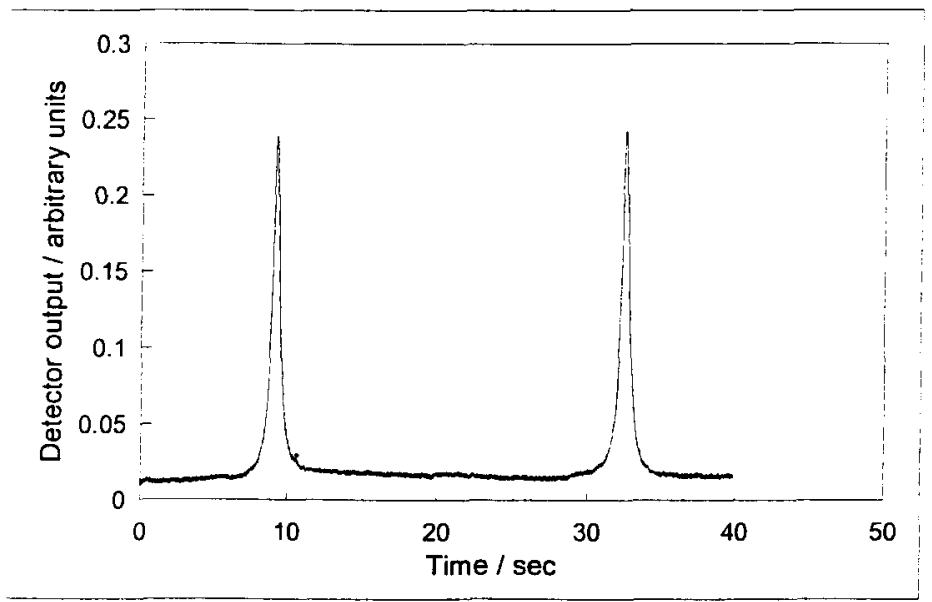

Figure 4. Finesse scan showing two orders of the $3.39 \mu \mathrm{m}$ HeNe laser.

\subsection{Scan Repeatability and Linearity}

Figure 5 shows eight scans of the $3.39 \mu \mathrm{m}$ line each of $10 \mathrm{sec}$ duration, and each covering two orders, superimposed to illustrate the scan repeatability of the system. An excellent degree of registration is achieved for the peaks recorded in scan mode, whilst those recorded on flyback, when the system is not controlled, are less well registered. The limit to measurement is the line thickness of the trace, suggesting an upper limit short term wavelength repeatability of better than $0.003 * \mathrm{FSR}$.

Figure 6 shows the cavity spacing of the etalon plotted against demand position on the CS100 interface. The residuals from the best straight line fit to the data points, shown on the right hand scale, suggest a non-linearity of better than $0.4 \%$. Moreover, this non-linearity is repeatable to better than can be measured and can therefore be corrected for in software if required.

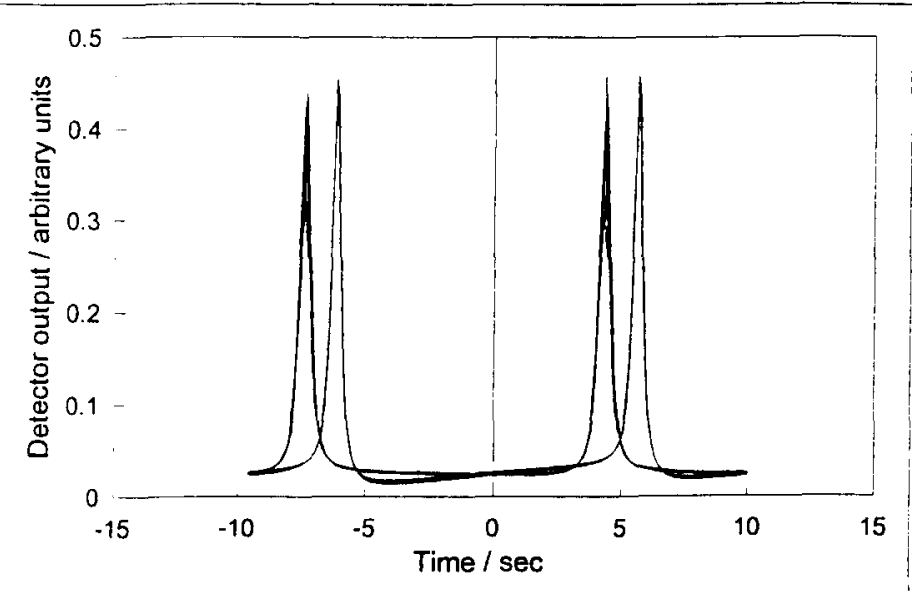

Figure 5. Overlay of eight scans of the $3.39 \mathrm{~mm}$ line, each covering two orders. 


\subsection{Response Time}

The effect of chopping the passband on and off the line is shown in Figure 7, suggesting a small signal response time of $30 \mathrm{msec}$. This compares with a response time of $2 \mathrm{msec}$ when the etalon is warm and at atmospheric pressure.

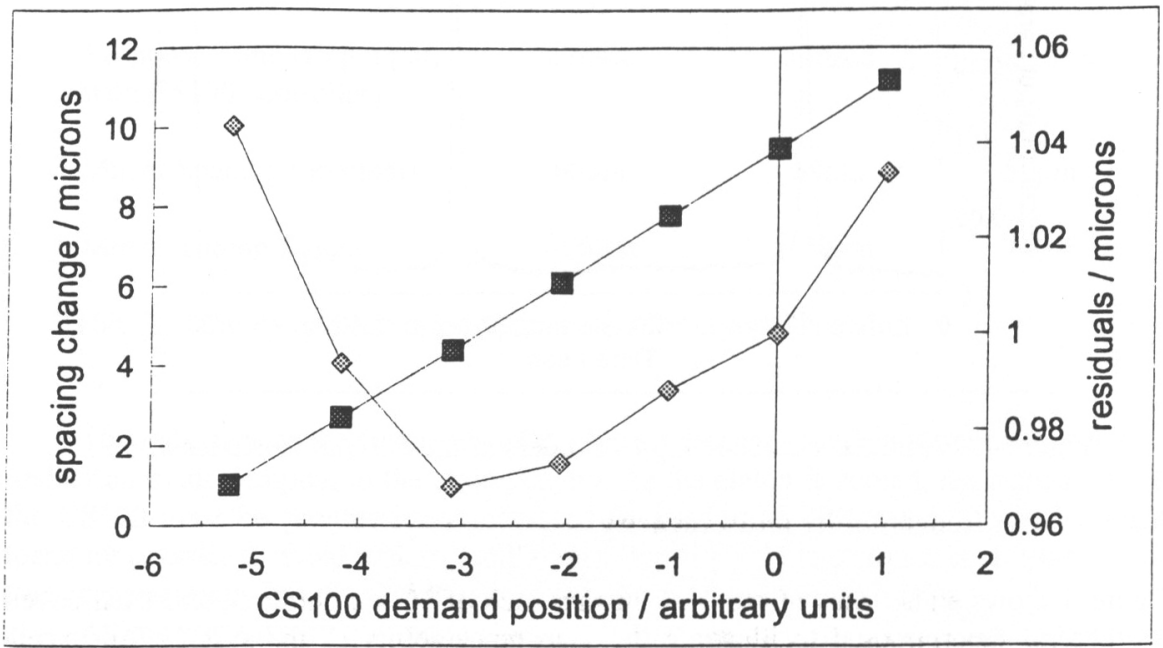

Figure 6. Etalon cavity length (left scale) vs. demand position on the CS100 interface. The residuals from the best straight line fit (right scale) indicate a linearity of better than $0.4 \%$. This is repeatable and therefore software correctable if required.

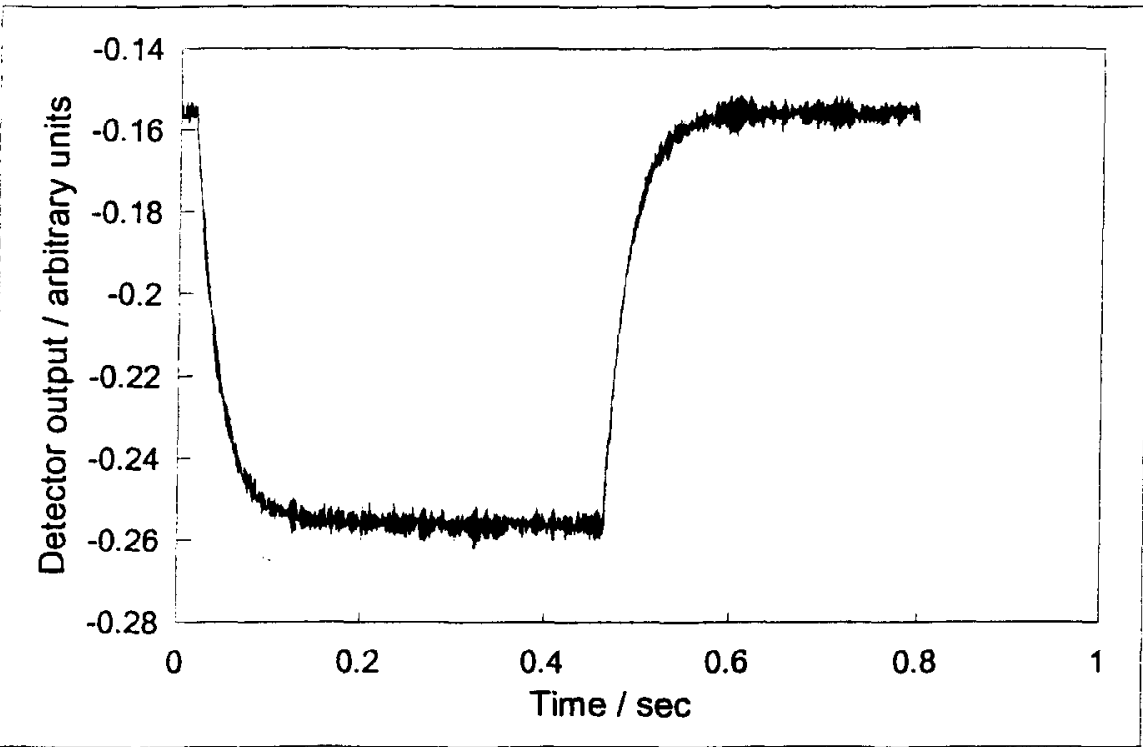

Figure 7. System response to step input (etalon at liquid nitrogen temperature). 


\section{Future Developments}

Development effort is now being focused on making the system space worthy. The capacitance bridge has now been hybridized in a space qualified package for the ESA-SILEX (Semiconductor Intersatellite Laser project) project. This device is the first step to reducing the size, weight and power requirement of the FP control electronics.

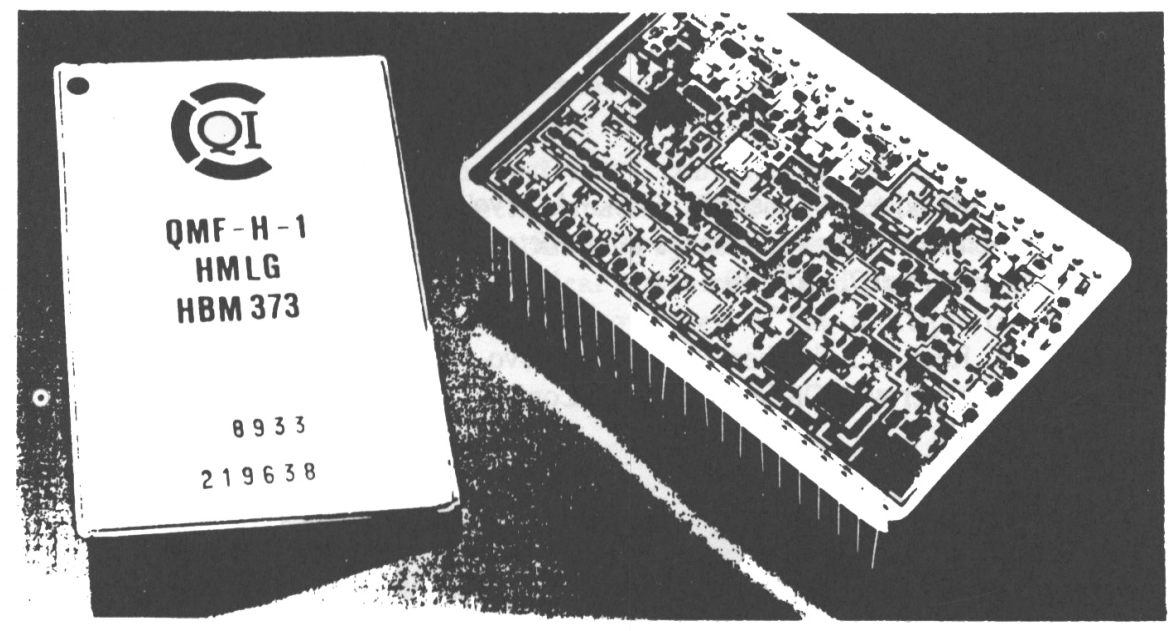

Figure 8. A capacitance bridge hybrid similar to that developed for the ESA-SILEX project. The hybrid is equivalent to two etalon servo-control channels, excluding the piezoelectric drives.

Ruggedization of the etalon is essential. Measures under consideration to improve robustness include using silicon mirrors for $3-5 \mathrm{~mm}$ systems. This would have the added benefit of reducing etalon weight and design complexity.

\section{Concluding Remarks}

With some qualifications (eg response time) we have succeeded in our objective of building a cryogenic etalon which can be stabilized using by the standard CS100 control unit. Response time could be improved with a redesign of the CS100 controller.

A similar etalon design is available for the $2-2.5 \mu \mathrm{m}$ region, using water-free fused silica substrates. and in principal the Zinc Selenide etalon described above can be adapted for the $9-13 \mu \mathrm{m}$ wavelength region using longer piezoelectric actuators

\section{Acknowledgments}

The authors wish to thank colleagues at Queensgate Instruments who have made contributions to the work reviewed above, in particular Dr. T. R. Hicks and C.M. Shannon. 
We gratefully acknowledge the support and advice, over a long period of time, of Dr. J. Fischer of the Naval Research Laboratory, and the assistance of Dr. Keith Thompson, also of the Naval Research Laboratory, in obtaining the test data shown in figures 4,5 and 6 . of this paper.

We also gratefully acknowledge the support for the cryogenic etalon project of Dr. Bob Ryan of the Northrop Grumman Corporation, and the assistance of Drs. Ryan and Nils Fonneland in obtaining the data shown in figure 7 of this paper. We also acknowledge Northrop Grumman for the loan of test equipment.

\section{References}

T.R. Hicks, N.K. Reay, \& R.J. Scaddan, 1974, "A servo-controlled Fabry-Perot interferometer using capacitance micrometers for error detection", J. Phys. E Sci.Instrum., vol 7, pp27-30

T.R. Hicks, N.K. Reay, C.L.Stephens, 1976, "A Servo-controlled Fabry-Perot Interferometer with On-line Computer Control", Astron.\& Astrophvsics, vol 51, pp367--374 